\title{
Predicting the thermal conductivity of pyrochlore and defect fluorite nuclear waste forms using experimental data and density functional theory simulations
}

TIMOTHY MICHAEL LEWIS CONNOR ${ }^{1}$, PIOTR KOWALSKI $^{2}$ AND SARAH C FINKELDEI ${ }^{1}$

${ }^{1}$ University of California, Irvine

${ }^{2}$ Forschungszentrum Jülich

Presenting Author: tmconnor@uci.edu

Predicting the thermal conductivity of pyrochlore and defect fluorite nuclear waste forms using experimental data and density functional theory simulations

Timothy Connor, Piotr Kowalski, and Sarah Finkeldei

Abstract:

$\mathrm{A}_{2} \mathrm{~B}_{2} \mathrm{O}_{7}$ pyrochlores are considered as potential matrices for permanent immobilization of $\mathrm{Pu}$ and minor actinides as one potential nuclear waste form for deep geological disposal. Over the long course of disposal, the waste forms will be subjected to self-irradiation from immobilized radionuclides. The so generated heat could influence the materials by causing cracking, altering corrosion resistance, or reducing phase stability. The ability of a waste form to effectively transfer heat, i.e. thermal conductivity is therefore a key parameter impacting the performance of nuclear waste forms in a deep geological repository [1]. Furthermore, the self-irradiation of the waste form will generate defects that can lead to an order/disorder transition from a pyrochlore to a defect fluorite structure. These structural changes are also expected to affect the thermal conductivity of pyrochlore-type waste forms. We used a combination of Density Functional Theory (DFT) and the Slack Model to calculate the intrinsic thermal conductivity of a series of $\mathrm{Nd}_{2-\mathrm{x}} \mathrm{Zr}_{2+\mathrm{x}} \mathrm{O}_{7 \pm \mathrm{y}}$ pyrochlores and defect fluorites with various $\mathrm{Nd} / \mathrm{Zr}$ ratios. We used this computed data to interpret thermal conductivity measurements obtained by Laser Flash Analysis. In particular, we were able to separate the intrinsic and extrinsic, i.e. defectrelated components using an approach developed by Tian et. al [2]. Knowledge of the changes in intrinsic and extrinsic thermal conductivity of pyrochlore and defect fluorite phases provides new insight into the evolution of heat transfer capabilities of pyrochlore-type nuclear waste forms during storage, and sheds light on the effect of atomic-scale ordering/disordering in these materials. This knowledge contributes to refining the understanding of the long-term performance of $\mathrm{Nd}_{2} \mathrm{Zr}_{2} \mathrm{O}_{7}$ pyrochlore-based waste forms.

\section{References:}

[1] D. Li, X. Sun, M. Khaheel. "Computationally Efficient Upscaling Methodology for Predicting Thermal Conductivity of Nuclear Waste Forms". DOE Report, Fuel Cycle and Research Development. 2011

[2] Z. Tian, L. Sun, J. Wang, J. Wang. "Theoretical prediction and experimental determination of the low lattice thermal conductivity of $\mathrm{Lu}_{2} \mathrm{SiO}_{5}$ ". Journal of the European Ceramic 